\title{
Polymerase ribozyme efficiency increased by G/T-rich DNA oligonucleotides
}

\author{
CHENGGUO YAO and ULRICH F. MÜLLER ${ }^{1}$ \\ Department of Chemistry and Biochemistry, University of California, San Diego, La Jolla, California 92093, USA
}

\begin{abstract}
The RNA world hypothesis states that the early evolution of life went through a stage where RNA served as genome and as catalyst. The replication of RNA world organisms would have been facilitated by ribozymes that catalyze RNA polymerization. To recapitulate an RNA world in the laboratory, a series of RNA polymerase ribozymes was developed previously. However, these ribozymes have a polymerization efficiency that is too low for self-replication, and the most efficient ribozymes prefer one specific template sequence. The limiting factor for polymerization efficiency is the weak sequence-independent binding to its primer/template substrate. Most of the known polymerase ribozymes bind an RNA heptanucleotide to form the P2 duplex on the ribozyme. By modifying this heptanucleotide, we were able to significantly increase polymerization efficiency. Truncations at the 3 '-terminus of this heptanucleotide increased full-length primer extension by 10 -fold, on a specific template sequence. In contrast, polymerization on several different template sequences was improved dramatically by replacing the RNA heptanucleotide with DNA oligomers containing randomized sequences of 15 nt. The presence of $G$ and $T$ in the random sequences was sufficient for this effect, with an optimal composition of $60 \% \mathrm{G}$ and $40 \% \mathrm{~T}$. Our results indicate that these DNA sequences function by establishing many weak and nonspecific base-pairing interactions to the single-stranded portion of the template. Such low-specificity interactions could have had important functions in an RNA world.
\end{abstract}

Keywords: RNA world; ribozyme; polymerase ribozyme

\section{INTRODUCTION}

According to the RNA world hypothesis, the early evolution of life went through a stage in which both the genome and genome-encoded catalysts were composed of RNA (Woese 1967; Crick 1968; Orgel 1968; Gilbert 1986). This hypothesis is supported by the identification of RNA molecular fossils in existing organisms and by the generation of artificial catalytic RNAs (ribozymes) that can catalyze a large diversity of chemical reactions. Additionally, the RNA world hypothesis can explain how an interdependent system of DNA and proteins in today's life forms could emerge from a simpler system (for reviews, see Muller 2006; Chen et al. 2007; Cheng and Unrau 2010). The central ribozyme in an RNA organism would have been an RNA polymerase ribozyme, to facilitate self-replication. To recapitulate an RNA world in the laboratory, 11 different RNA polymerase ribozyme variants were developed previously (Johnston et al. 2001; Lawrence 2005; Zaher and

\footnotetext{
${ }^{1}$ Corresponding author.

E-mail ufmuller@ucsd.edu.

Article published online ahead of print. Article and publication date are at http://www.rnajournal.org/cgi/doi/10.1261/rna.2726811.
}

Unrau 2007; Wochner et al. 2011). One of them made it possible to extend a small fraction of the primer by $95 \mathrm{nt}$, by base-pairing the ribozyme 5 '-terminus to the template, and by using a template composed of 11-nt repeats, each of them interacting favorably with the ribozyme (Wochner et al. 2011). However, these constraints on the template sequence would make it difficult or impossible to obtain a self-replicating polymerase ribozyme. Therefore, our task is to improve the sequence-independent binding of the primer/template by the ribozyme.

Our study focuses on the earliest variants of this ribozyme, Polle ("Evolved Pol1") (Johnston et al. 2001; Lawrence and Bartel 2005) and its close relative, B6.61 (Zaher and Unrau 2007), because these two ribozymes show the highest polymerization efficiency among the first 10 discovered ribozymes. Although the eleventh ribozyme has a higher polymerization efficiency (Wochner et al. 2011), it shares with B6.61 and Polle the same problem of weak sequenceindependent substrate binding. Therefore, our study uses two of the most efficient polymerase ribozymes, to address a problem common to all existing polymerase ribozymes.

The ribozyme Polle requires the heptanucleotide cofactor 5'-GGCACCA for optimal polymerization efficiency 
(Fig. 1; Johnston et al. 2001). This heptanucleotide is also called the P2 oligo because it is required to form the P2 stem on the ribozyme. This P2 stem served to base-pair the substrate to the catalytic core, in a cis-acting ancestor of the ribozyme (Ekland and Bartel 1995). In the trans-acting polymerase ribozyme, the $\mathrm{P} 2$ stem is not connected to the substrate, but it may serve in a structural role near the catalytic site. In contrast to Polle, the ribozyme B6.61 functions best in the absence of the P2 oligo. This difference is probably caused by four additional nucleotides at the 5 '-terminus of B6.61, which appear to interact with the same sequence that is targeted by the P2 oligo (Zaher and Unrau 2007). Because the P2 oligo is positioned adjacent to the catalytic site on the ribozyme, we hypothesize that modifying the P2 oligo in its length and its sequence could lead to ribozymes with higher polymerization efficiency and lower dependence on specific template sequences.

\section{RESULTS}

As a starting point for our study, we recapitulated the previously known influence of the wild-type $\mathrm{P} 2$ oligo on ribozyme polymerization (Fig. 2A). In the absence of a P2 oligo, the polymerase ribozyme rapidly extended the primer on template T21 by 5 or 6 nt but then stalled at this position, preventing full-length extension. In the presence of the wild-type RNA P2 oligo (5'-GGCACCA), this stalling was reduced and allowed $3 \%$ of the primers to be extended to full length. This is consistent with previous analyses (Johnston et al. 2001; Lawrence and Bartel 2003).

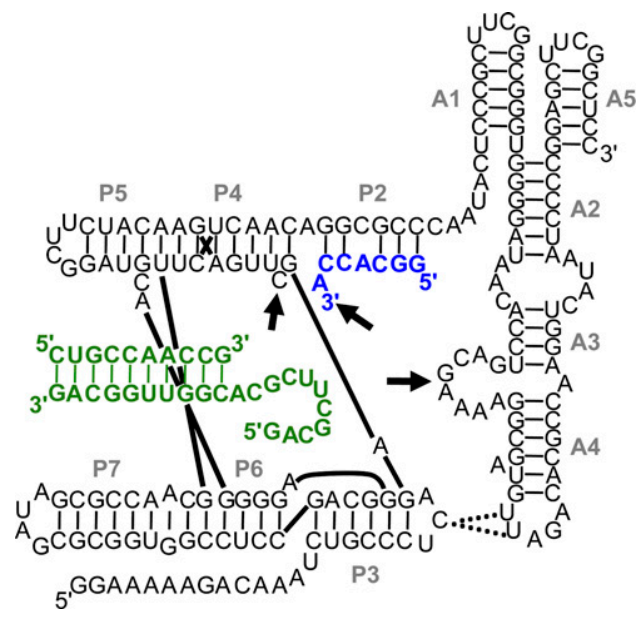

FIGURE 1. Secondary structure of the polymerase ribozyme Polle. The secondary structure is based on Johnston et al. (2001), Shechner et al. (2009), and Wang et al. (2011). (Blue) The wild-type P2 oligo; (green) the primer/template substrate with the sequence of template T21 and the corresponding primer. (Arrowheads) Residues close to the catalytic site. These are the unpaired C in helix P4 (Shechner et al. 2009), the 3 '-terminus of the P2 oligo (Ekland and Bartel 1996), and the loop connecting helices A3 and A4 (Wang et al. 2011). (Dotted lines) The tertiary contacts between the accessory domain and ligase core (Wang et al. 2011).

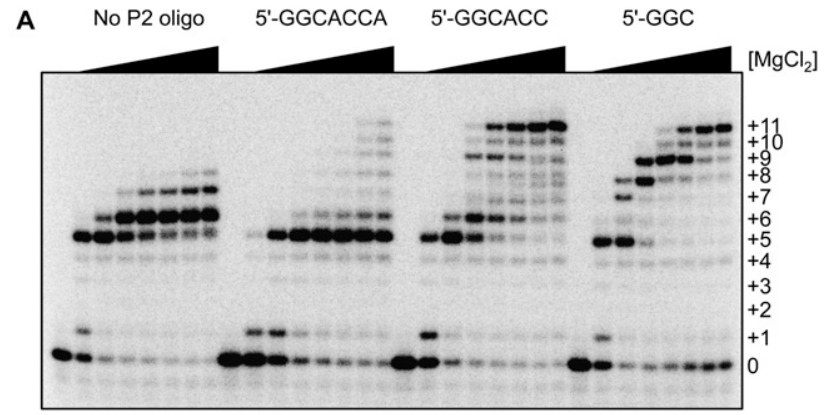

B

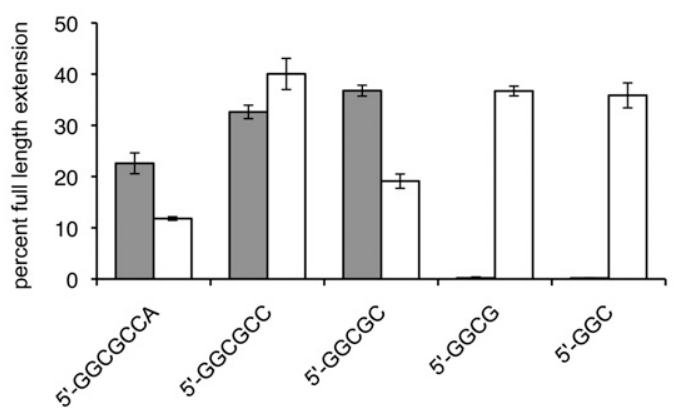

FIGURE 2. Effect of $\mathrm{P} 2$ oligo truncations on polymerization efficiency, with template T21. (A) Autoradiogram of polymerization products after PAGE separation. The concentrations of free magnesium correspond to the total magnesium concentration minus the concentration of NTPs $(16 \mathrm{mM})$. The total magnesium concentration ranged from $20 \mathrm{mM}$ to $200 \mathrm{mM}$. The number of nucleotides added to the primer are shown on the right. (B) Polymerization efficiency with the wild-type P2 oligo and 3'-truncated P2 oligos. The polymerization efficiency was quantified as the percentage of primers that were extended to full length of the template. Results for RNA P2 oligos (white) and DNA P2 oligos (gray) are shown. Error bars denote standard deviations from three experiments. A list with the efficiencies of more truncated P2 oligos is given in Supplemental Table S1.

\section{Truncations of P2 oligonucleotides}

To test whether truncations of the P2 oligonucleotide could improve polymerization efficiency, we generated a series of RNA P2 oligonucleotides and tested their effects in ribozyme polymerization assays. When the 3 '-terminal adenosine was removed from the wild-type P2 oligo, $40 \%$ of the primers were extended to full length, a more than 10 -fold improvement over the wild-type P2 oligo. With this truncation, stalling at nucleotides +5 and +6 was almost undetectable at high magnesium concentrations (Fig. 2A). Truncations at the $3^{\prime}$-terminus of the RNA P2 oligo maintained most of the activity down to a length of $3 \mathrm{nt}$. Dinucleotides did not benefit polymerization, and truncations on the $5^{\prime}$-terminus abolished the beneficial effect of the P2 oligo (Supplemental Table S1). To strengthen the weaker DNA/RNA duplex, we converted the internal, mismatching A of DNA P2 oligos to a matching G. With this mutation, DNA P2 oligos caused similar effects as the RNA P2 oligos (Fig. 2B). These results showed that RNA and DNA P2 oligos had increased efficiency on template T21 when their $3^{\prime}$-terminal nucleotides were removed. 
Why does the P2 oligo $3^{\prime}$-terminus stall polymerization (Fig. 2A)? We hypothesized that the $3^{\prime}$-terminus of the P2 oligo interacts with $2^{\prime}$-hydroxyl groups in the singlestranded portion of the template, as suggested by the crystal structure of the ligase (Shechner et al. 2009) and by studies on template 2'-hydroxyl groups (Müller and Bartel 2003). This hypothesis was confirmed when we found that the effects of 2 '-deoxy mutations on polymerization efficiency were abolished by truncating 1 or $4 \mathrm{nt}$ from the wild-type P2 oligo (Fig. 3). Therefore, the stalling on template T21 is caused by direct or indirect interactions between the $3^{\prime}$ terminus of the wild-type P2 oligo and specific 2 '-hydroxyl groups (or a modified sugar pucker) of the template.

The beneficial effect of truncated P2 oligos was stronger with the polymerase ribozyme Polle than with the ribozyme B6.61 (Supplemental Table S2). Therefore, our study focused on the polymerase ribozyme Polle. The beneficial effects of truncated P2 oligos were also weaker when three different, newly designed template sequences (T50a, T50b, T50c) were used instead of template T21 (data not shown). Therefore, the beneficial effects of truncated P2 oligos were dependent on the context of ribozyme variant and template. Specifically, these P2 oligos worked best in the context in which the polymerase ribozyme interacts efficiently with the primer/template, i.e., Polle with template T21.

The trinucleotide 5'-GGC improved polymerization on all four templates (Fig. 4). Because random trinucleotides may have been abundant in an RNA world, we tested

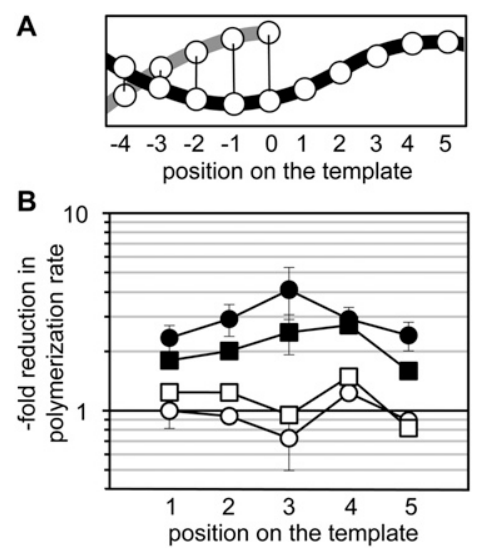

FIGURE 3. Effect of $2^{\prime}$-deoxy modifications in the template on polymerization efficiency, in the context of truncated P2 oligos. $(A)$ Numbering of $2^{\prime}$-hydroxyl groups relative to the $3^{\prime}$-terminal nucleotide of the primer. (B) Quantitation of $2^{\prime}$-deoxy effects in the presence of the RNA P2 oligos 5'-GGCACCA (filled circles), 5'GGCACC (filled squares), 5'-GGC (open squares), or in the absence of a $\mathrm{P} 2$ oligo (open circles). The graph shows the fold reduction in the rate of primer extension when the $2^{\prime}$-hydroxyl group at a given position in the template was replaced by a $2^{\prime}$-deoxy modification. The difference in the $2^{\prime}$-deoxy effects between P2 oligos $5^{\prime}$-GGCACCA and $5^{\prime}$-GGC shows that the $2^{\prime}$-deoxy effect is caused by the $3^{\prime}$ terminal sequence "ACCA." Error bars are standard deviations of three independent experiments. Error bars smaller than the symbol are hidden by the symbol.

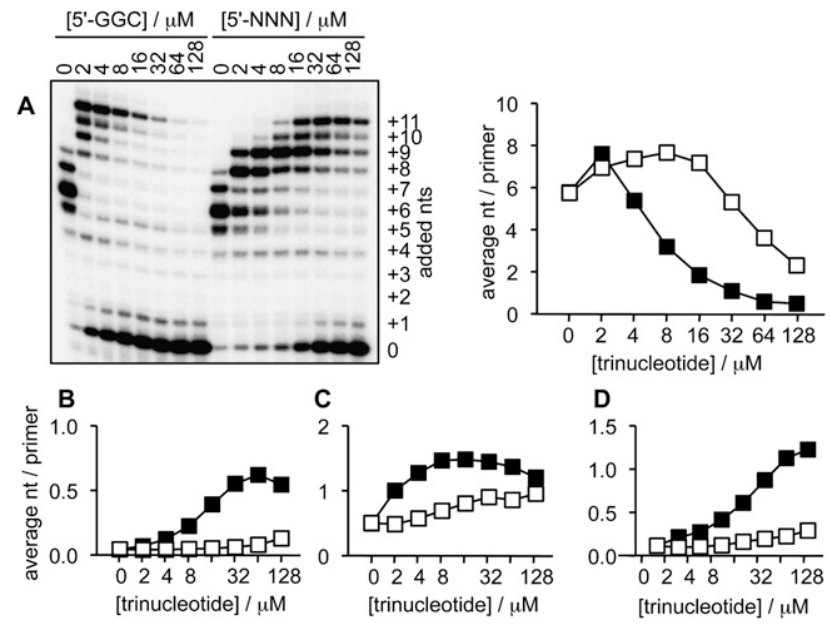

FIGURE 4. Effect of trinucleotide RNA P2 oligos on polymerization efficiency with four different templates. For template T21 $(A)$, a representative autoradiogram of PAGE-separated polymerization products and its quantitation is shown. The concentrations of the trinucleotides $(0 \mu \mathrm{M}$ to $128 \mu \mathrm{M})$ and the number of added nucleotides (nts) are indicated on the autoradiogram. For the other templates, $(B)$ template T50a, $(C)$ template T50b, and $(D)$ template T50c, only the quantitation is shown. The quantitations show a plot of the average number of nucleotides added per primer as a function of the concentration of the trinucleotides $5^{\prime}$-GGC (filled squares) or $5^{\prime}$-NNN (open squares). Standard deviations of triplicate experiments were smaller than the symbols in the graph, typically in the range of $0.03 \mathrm{nt} /$ primer.

whether the polymerase ribozyme would be able to select the cognate trinucleotide from a mixture of random trinucleotides. A mixture of random trinucleotides contains 64 different sequences. To allow the ribozyme to specifically use one sequence in this mixture, we titrated the trinucleotide up to the 64-fold stoichiometry of the ribozyme. For template T21, the P2 oligo $5^{\prime}-\mathrm{NNN}$ facilitated the full stimulatory effect of the P2 oligo $5^{\prime}$-GGC, although at a fourfold excess over the ribozyme (Fig. 4). Analogously, the other three templates also benefited from both $\mathrm{P} 2$ trinucleotides but required higher concentrations of $5^{\prime}-\mathrm{NNN}$ than of $5^{\prime}$-GGC for the same effect. Higher concentrations of either trinucleotide inhibited polymerization with the template T21 but not significantly with the other templates. These results showed that the polymerase ribozyme could select beneficial sequences out of a random pool of trinucleotides and use them to improve its polymerization efficiency.

\section{Extensions of P2 oligonucleotides}

In our search for extensions of P2 oligos that increase polymerization efficiency, we used DNA oligonucleotides because of the prohibitive cost for a large number of RNA oligonucleotides. All P2 oligos used the 5 '-terminal sequence $5^{\prime}$-GGCGCC. We were surprised to find that high increases in polymerization efficiency were caused by $3^{\prime}$-extensions with randomized sequences (data not shown). This effect 
was detectable with a length of 10 randomized nucleotides and saturated with 15-20 randomized nucleotides. An RNA P2 oligo with the same sequence ( $5^{\prime}$-GGCGCCN 15 ) did not benefit polymerization. To determine which nucleotides in the randomized sequence were required for the increased polymerization efficiency, we tested DNA P2 oligos with subsets of nucleotides in their $3^{\prime}$-terminal randomized sequence (Fig. 5; Supplemental Table S3). Specifically, we compared the polymerization efficiencies when the randomized portion of the extended P2 oligos contained the four homopolymers, all six combinations of two nucleotides, all four combinations of three nucleotides, and all four nucleotides. The four best compositions of random sequences were $(\mathrm{G} / \mathrm{T})_{15},(\mathrm{~A} / \mathrm{C} / \mathrm{G} / \mathrm{T})_{15},(\mathrm{C} / \mathrm{G} / \mathrm{T})_{15}$, and $(\mathrm{A} / \mathrm{G} / \mathrm{T})_{15}$. These compositions coincide with the only four compositions that contain both $\mathrm{G}$ and $\mathrm{T}$. We focused our study on P2 oligos with the sequence $5^{\prime}$-GGCGCC $(\mathrm{G} / \mathrm{T})_{15}$ to simplify the investigation described below.

To determine specific sequences of Gs and Ts that promoted high polymerization activity, we tested a series of arbitrarily chosen $5^{\prime}$-GGCGCC $(\mathrm{G} / \mathrm{T})_{15}$ sequences (Supplemental Table S4). Comparison of their effects on polymerization efficiency suggested that a guanosine was the

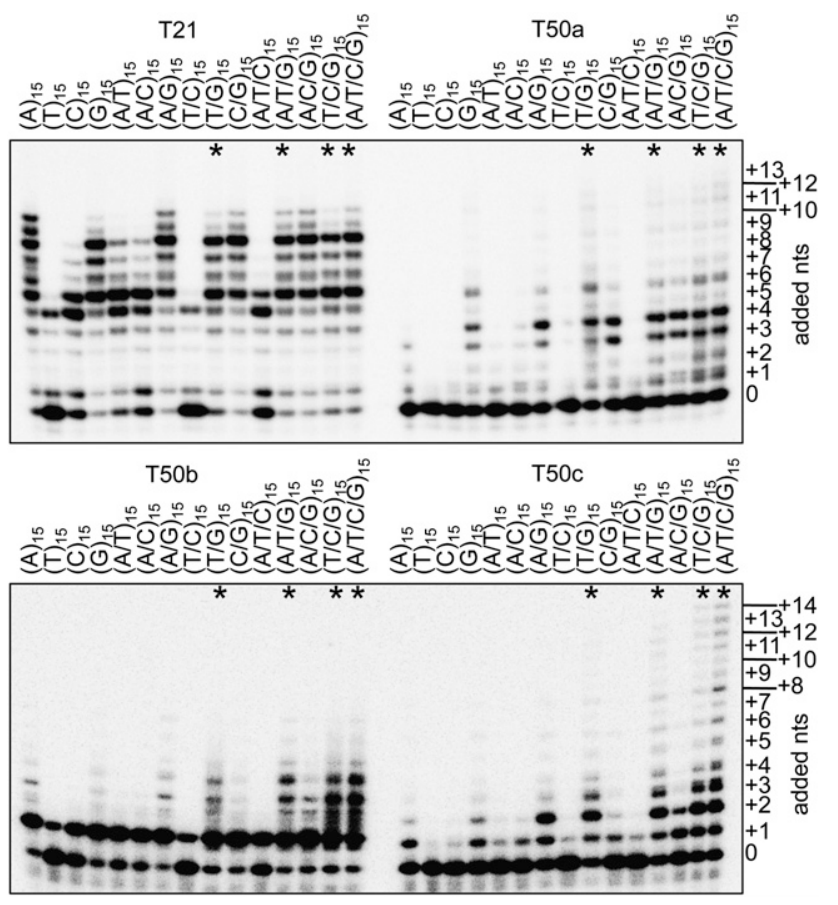

FIGURE 5. Effect of the nucleotide composition of 15-nt randomized DNA P2 oligo 3 '-extensions on polymerization efficiency. Representative autoradiograms of PAGE-separated polymerization products are shown, for reactions with the four different templates T21, T50a, T50b, and T50c. Only the $3^{\prime}$-extensions to the DNA P2 oligo sequence $5^{\prime}$-GGCGCC are shown. The number of nucleotides added to the primer during polymerization is indicated. Asterisks mark the four P2 oligos that mediated the highest polymerization efficiency, averaged over all templates. The values of polymerization efficiency are given in Supplemental Table S3. preferred first nucleotide and that the activity was highest when the $3^{\prime}$-extension contained $60 \% \mathrm{G}$ and $40 \% \mathrm{~T}$ (Fig. 6; Supplemental Table S4). This corresponds to 9 G's and 6 T's in the 15 -nt-long $3^{\prime}$-extension. To identify sequence motifs that correlate with high activity, we generated 20 additional 3 '-extensions with arbitrary sequences of 9 G's and 6 T's and tested them for their activity. However, sequence alignments did not show clustering of sequences with high activity (data not shown), and no correlation was found between a specific sequence motif and high or low activity.

How could the pools of P2 oligos with randomized 3'extensions cause high polymerization efficiency although many of the individual sequences did not confer high activity? To answer that question, we measured the effects of three DNA P2 oligos separately and in a reaction mixture (Fig. 7A). The individual P2 oligos mediated high, medium, or low polymerization efficiency $(4.7 \pm 0.2,2.08 \pm 0.02$, and $0.37 \pm 0.03 \mathrm{nt} /$ primer, respectively). However, the polymerization efficiency of a reaction mixture containing all three P2 oligos was $3.3 \pm 0.2 \mathrm{nt} /$ primer, significantly higher than the average from the individual reactions $(2.37 \pm 0.07 \mathrm{nt} /$ primer). We assume that inefficient $\mathrm{P} 2$ oligos do not bind the templates, while efficient P2 oligos that bind the templates will increase the polymerization efficiency. This can explain why pools of P2 oligos with randomized 3'extensions can improve polymerization almost as efficiently as the best P2 oligos with specific sequences.

To illustrate the dramatic effects of specific P2 oligos, we compared the polymerization efficiencies of the ribozyme Polle with the original RNA P2 oligo 5'-GGCACCA, to the ribozyme $\mathrm{B} 6.61$, the ribozyme Polle with the $3^{\prime}$-truncated DNA oligo 5'-GGCGCC, and the ribozyme Polle with the most efficient G/T-rich DNA P2 oligo (Fig. 7B). The first three scenarios did not extend the majority of the primers by $>2$ nt. However, the G/T-rich P2 oligo mediated efficient polymerization, and primers elongated by $19 \mathrm{nt}$ were detectable. This finding is significant because it shows that P2 oligos with G/T-rich $3^{\prime}$-extensions can facilitate efficient polymerization on new template sequences and thereby decrease the current dependence of the polymerase ribozyme on variants of the template T21.

An earlier study suggested that the wild-type P2 oligo increases polymerization efficiency at the cost of polymerization fidelity (Zaher and Unrau 2007). Therefore, we tested whether the polymerization fidelity changed in the presence of $(\mathrm{N})_{15}$ or $(\mathrm{G} / \mathrm{T})_{15} 3^{\prime}$-extensions on the P2 oligos (Fig. 8). The fidelity was highest on the two most efficient templates, T21 and T50c. Only on template T21 was it possible to compare all P2 oligos for the addition of all NTPs. Here, the fidelity was higher for the wild-type P2 oligo than for the P2 oligos with a randomized or G/T-rich 3 '-extension. On the other templates, the fidelity was similar with and without the extensions, or could not be determined due to lower polymerization efficiency with the wild-type $\mathrm{P} 2$ oligo. Therefore, our results suggest that an increase in polymerization 


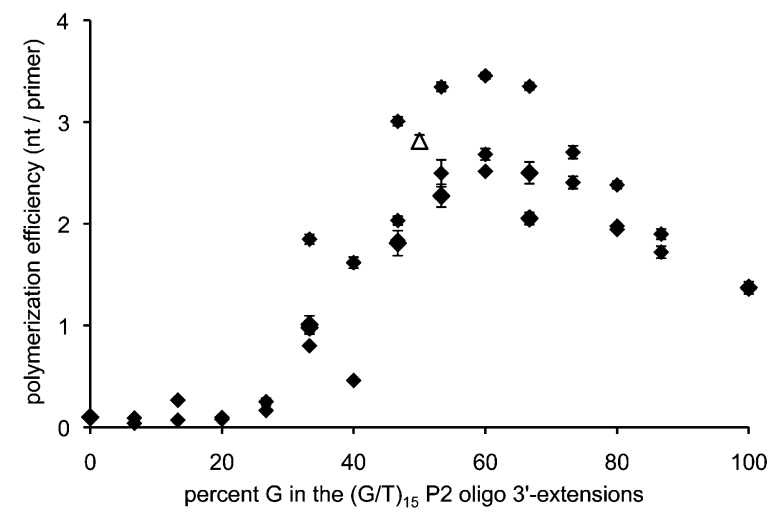

FIGURE 6. Optimal nucleotide bias in $(\mathrm{G} / \mathrm{T})_{15}$ P2 oligo $3^{\prime}$-extensions. The polymerization efficiency is shown as a function of the percentage of guanosines in the $3^{\prime}$-extensions to the DNA P2 oligo sequence 5'-GGCGCC. Extensions with defined sequences (filled diamonds) or a randomized sequence with $50 \%$ G (open triangle) were investigated. The values for polymerization efficiency are the geometric means for polymerization efficiencies with the templates T21, T50a, T50b, and T50c. Standard deviations of triplicate experiments are shown as error bars but are often smaller than the symbols in the graph. The P2 oligo sequences and the values of polymerization efficiency are given in Supplemental Table S4.

efficiency with G/T-rich P2 oligos was partially bought by a decrease in polymerization fidelity.

What is the mechanism of G/T-rich $3^{\prime}$-extensions increasing polymerization efficiency? The single-stranded portion of the template and the 3 -extension of the P2 oligo are in close proximity, as judged by the crystal structure of the ribozyme catalytic core (Shechner et al. 2009), the covalent connection of the template sequence and P2 oligo in the precursor ribozyme (Ekland and Bartel 1995), and the effects of $2^{\prime}$-deoxy modifications in the template (Fig. 3; this study). This suggests that these two single-stranded sequences may be base-pairing to each other (Fig. 9A). To test the hypothesis that such base-pairing increases polymerization efficiency, we plotted the polymerization efficiency as a function of the computationally predicted base-pairing stability between the single-stranded portion of the templates and the P2 oligo 3'-extensions (Fig. 9B). The 23 DNA P2 oligos with $60 \% \mathrm{G}$ and $40 \% \mathrm{~T}$ were used for this comparison. The correlations on the three templates T50a, T50b, and T50c confirmed the hypothesis. In contrast, template T21 does not generate such a correlation. This is consistent with the lack of beneficial effects of DNA P2 oligo $3^{\prime}$-extensions on template T21 (cf. Figs. 2A and 5), and is probably a result of specific interactions between the polymerase ribozyme and template T21 (Lawrence and Bartel 2003).

\section{DISCUSSION}

In this study, we found that modifications of the RNA P2 oligos can strongly increase the polymerization efficiency of the polymerase ribozyme Polle. The P2 oligo truncations act in a very different fashion from the $\mathrm{P} 2$ oligo extensions.
The truncations removed an inhibitory interaction of the P2 oligo 3'-terminus with template T21. Correspondingly, truncations at the P2 oligo $3^{\prime}$-terminus led to strong improvement only on template $\mathrm{T} 21$. In contrast, converting the P2 oligos to DNA and extending their 3 '-terminus by $(\mathrm{G} / \mathrm{T})_{15}$ sequences caused dramatic improvements in polymerization efficiency on three completely unrelated templates. This effect reached an optimum when the $3^{\prime}-$ extension contained $60 \% \mathrm{G}$ and $40 \% \mathrm{~T}$ residues.

How can these $(G / T)_{15}$ sequences increase polymerization efficiency? We propose that the increased polymerization efficiency lies in a general property of these sequences: Their composition could enable them to form imperfect duplexes with a broad diversity of template sequences. The guanosines in the P2 oligos could base-pair to both $\mathrm{C}$ and $\mathrm{U}$, and the thymidines could base-pair to both A and to $\mathrm{G}$ (Hunter et al. 1987; Kalnik et al. 1988). Therefore, we propose that the P2 oligo 3'-extensions may form weak base pairs of low sequence specificity with the template sequence. Such basepairings would increase the substrate binding affinity, and the weakness and low sequence specificity could explain why these effects are functional on different template sequences. These interactions would be weak enough to allow a repositioning on the template because the predicted duplexes were never longer than $5 \mathrm{bp}$, and our study showed that a different DNA/RNA duplex required at least five G:C base pairs for
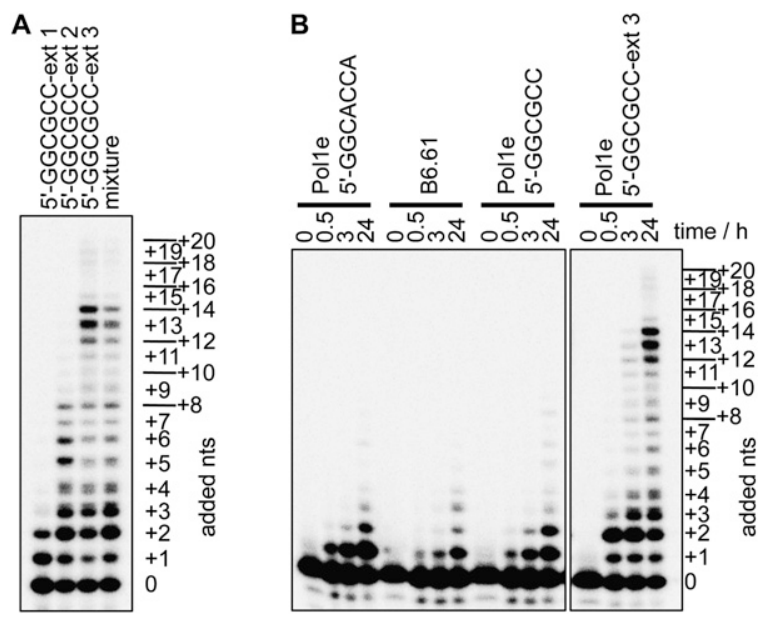

FIGURE 7. Effects of P2 oligo extensions on polymerization efficiency. Autoradiograms of PAGE-separated polymerization products are shown, for reactions with the template T50c. (A) Comparison between three separate reactions with three different P2 oligos containing the $3^{\prime}$-extensions ext 1 , ext2, and ext 3 , and a reaction containing the same three $\mathrm{P} 2$ oligos in one reaction mixture. The number of nucleotides (nts) that were added to the primer is indicated. The polymerization efficiencies are given in the text, with errors as standard deviations from triplicate experiments. $(B)$ Comparison of four ribozyme/P2 oligo contexts. The polymerase ribozyme Polle with the wild-type RNA P2 oligo 5'-GGCACCA is compared to ribozyme B6.61 without $\mathrm{P} 2$ oligo, polymerase ribozyme Polle with the truncated DNA oligo 5'-GGCGCC, and polymerase ribozyme Polle with DNA P2 oligo 5'-GGCGCC-ext3. The polymerization reaction times are indicated. 


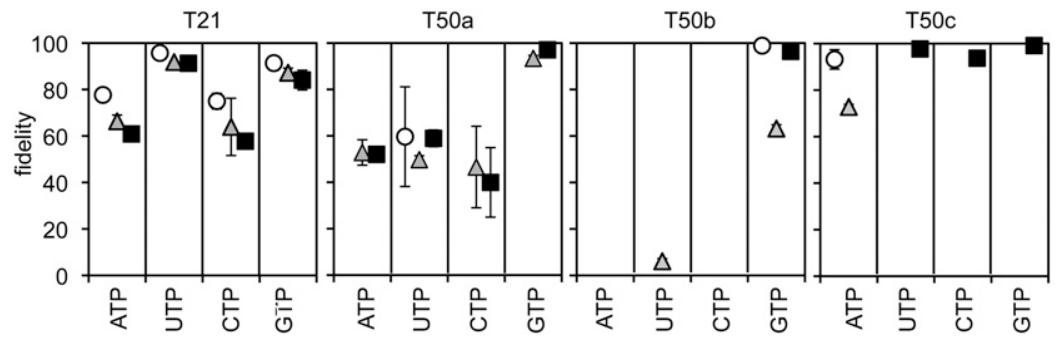

FIGURE 8. Influence of $\mathrm{P} 2$ oligo extensions on polymerization fidelity. The fidelity was calculated as the percent incorporation of the templated nucleotide. Four templates were used, T21, T50a, T50b, and T50c. The symbols denote the wild-type RNA P2 oligo (white circles), the DNA P2 oligo with extension $(\mathrm{N})_{15}$ (gray triangles), and the DNA P2 oligo with the most efficient $(\mathrm{G} / \mathrm{T})_{15}$ extension (ext3; black squares). Missing symbols indicate that the values could not be obtained due to low polymerization efficiency with the specific $\mathrm{P} 2$ oligo/template combinations. Errors are standard deviations from triplicate experiments, and are smaller than the symbol when they are not visible.

being functional under polymerization conditions (Fig. 2B). This would allow repositioning of the P2 oligo-template interactions after every few nucleotides, and thereby facilitate the observed polymerization of $19 \mathrm{nt}$.

One major obstacle for the emergence of an RNA world is the chemical diversity from which it must have arisen (Joyce 2002). Our study identified an example where chemical diversity could have been beneficial to the functioning of an RNA world. Noncanonical nucleobases that form base pairs with low fidelity could have provided the RNA world with a means to bind RNA sequences via their bases in a nonsequence-specific fashion. Thus, a goal for future research is to test whether noncanonical nucleobase analogs could be used to improve the efficiency of ribozyme polymerization.

The emergence of an RNA world assumes the prior existence of RNA polymers, created either by abiotic processes or by a pre-RNA world. We assume that trinucleotide sequences were present because short oligomers are usually abundant in reaction mixtures that generate RNA polymers generated under presumed prebiotic conditions (Huang and Ferris 2003). Trinucleotides with a random sequence may have been especially abundant, depending on the mechanism of synthesis. Our results showed that the polymerase ribozyme is able to select cognate $\mathrm{P} 2$ oligos from a random mixture of trinucleotides to improve its polymerization efficiency. Analogously, in an RNA world, multiple different ribozymes could have used the same diverse pool of trinucleotides, each ribozyme selecting its cognate cofactors.

\section{MATERIALS AND METHODS}

Polymerase ribozymes were synthesized by in vitro transcription with T7 RNA polymerase, from PCR products that included the T7 promoter (Johnston et al. 2001; Zaher and Unrau 2007). Transcription products were purified by denaturing PAGE. RNA oligonucleotides were purchased from Dharmacon/Thermofisher, deprotected, and purified by denaturing PAGE. RNAs shorter than $5 \mathrm{nt}$ were not PAGE-purified due to the high loss and because almost all of these short RNAs were full-length. We confirmed that the absence of gel purification had no effect on the polymerizations for these short RNAs. RNAs longer than $30 \mathrm{nt}$ were synthesized by in vitro transcription with T7 RNA polymerase, from annealed DNA oligonucleotides that included the T7 promoter, and purified by denaturing PAGE. DNAs were purchased from IDT or Valuegene. Random segments were generated by IDT, using machine mixing of phosphoramidites. DNAs were purified by PAGE if the length was $>30 \mathrm{nt}$.

Ribozyme polymerization reactions were performed as described previously (Johnston et al. 2001). If not indicated otherwise, RNAs were dissolved in water, heated for $2 \mathrm{~min}$ at $80^{\circ} \mathrm{C}$, and cooled to $17^{\circ} \mathrm{C}$ at $0.1^{\circ} \mathrm{C}$ per second. Reactions were started by adding reaction buffer so that the final concentrations were $200 \mathrm{mM}$ magnesium chloride, $50 \mathrm{mM}$ Tris/ $\mathrm{HCl}(\mathrm{pH} 8.5)$, and $4 \mathrm{mM}$ each NTP. For the magnesium titration in Figure 2A, the total magnesium concentration ranged from $20 \mathrm{mM}$ to $200 \mathrm{mM}$. The final concentrations of RNAs were $2 \mu \mathrm{M}$ ribozyme, $2.5 \mu \mathrm{M}$ P2 oligo, $1 \mu \mathrm{M}$ template, and traces of $5^{\prime}-\left[{ }^{32} \mathrm{P}\right]$ radiolabeled primer. Reactions were incubated for $24 \mathrm{~h}$ at $17^{\circ} \mathrm{C}$. The reactions were stopped by adding 1.5 volumes of a buffer containing $8 \mathrm{M}$ urea, $140 \mathrm{mM}$ $\mathrm{Na}_{2}$ EDTA, and $13 \mu \mathrm{M}$ an RNA oligonucleotide that was complementary to the template. The stopped reaction was heated for $2 \mathrm{~min}$ at $80^{\circ} \mathrm{C}$ and cooled to room temperature at $0.1^{\circ} \mathrm{C}$ per second. Samples were separated on $20 \%$ polyacrylamide $7 \mathrm{M}$ urea $0.5 \times \mathrm{TBE}$ gels. Autoradiograms of the separations were scanned by a PhosphorImager (Bio-Rad, PMI) and quantitated using the software Quantity One. The values for "nucleotides per primer" were obtained by multiplying the fraction of each band per lane with the number of added nucleotides corresponding to that band (Müller and Bartel 2003). For both ribozymes, the optimal reaction temperatures were at or near $17^{\circ} \mathrm{C}$. The exceptions were reactions with short DNA P2 oligos that preferred the lower reaction temperature of $12^{\circ} \mathrm{C}$, probably due to lower duplex stability. Additionally, the shortest RNA P2 oligos (3 or $4 \mathrm{nt}$ ) inhibited both tested polymerase ribozymes at temperatures below $17^{\circ} \mathrm{C}$.

The sequences of templates T21, T50a, T50b, and T50c were

5'-GACGCUUCGCACGGUUGGCAG-3', 5'-GAACUGACUGCCGAAUGUUUAAUGACCGUGACCCGUA CUACGGUUGGCAG-3',

5'-GAGCUUCGCCACUGUGUUAUACCGAGAUGUCAACCGU AACCGGUUGGCAG- ${ }^{\prime}$, and

5'-GGCAUACGAUGAUACGACCUAAGGCACUUUGACACCGU UUCGGUUGGCAG-3',

respectively. The sequence of the $5^{\prime}$-radiolabeled primer in all reactions was $5^{\prime}$-CUGCCAACCG- $3^{\prime}$. The sequences of the three DNA P2 oligos whose effects on polymerization efficiency were determined separately and in a mixture were

5'-GGCGCCGGGGTTTTTGTGGGG (5'-GGCGCC-ext1; $0.37 \pm$ $0.03 \mathrm{nt} /$ primer),

5'-GGCGCCGGGTTGGGTGTGTTG (5'-GGCGCC-ext2; $2.08 \pm$ $0.02 \mathrm{nt} /$ primer), and

5'-GGCGCCGGGTGTGGGGTTGTT (5'-GGCGCC-ext3; $4.7 \pm$ $0.2 \mathrm{nt} /$ primer). 
A
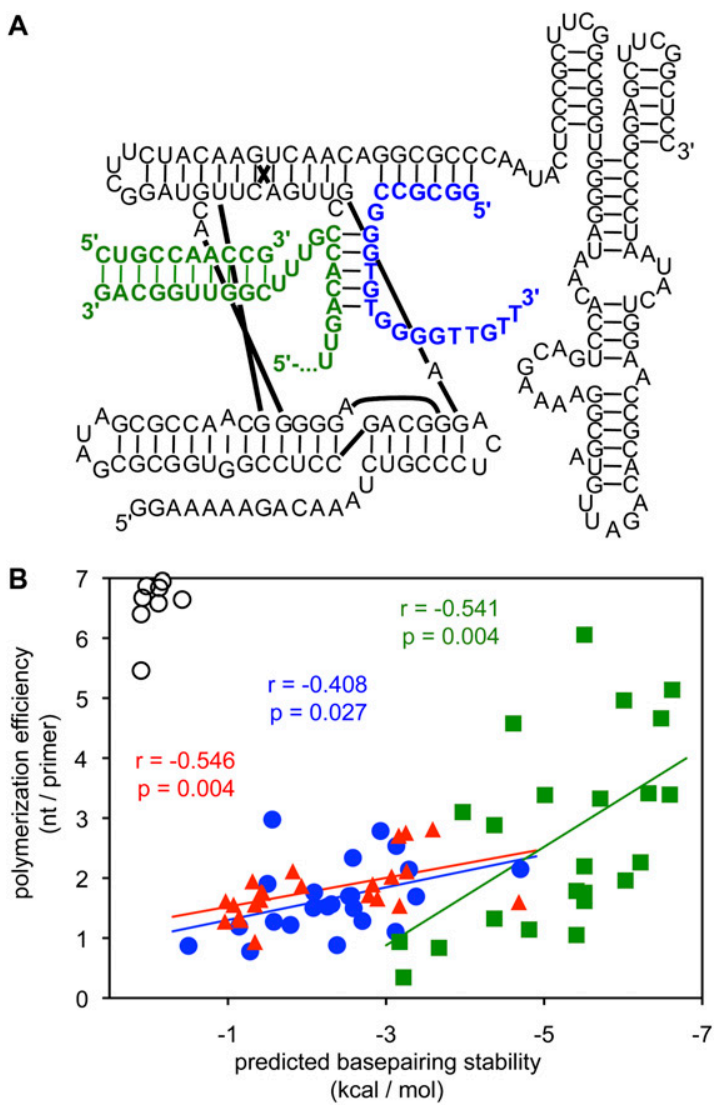

FIGURE 9. Proposed mechanism for substrate binding with the help of G/T-rich DNA P2 oligo extensions. (A) Secondary structure of the polymerase ribozyme Polle (black) with the proposed interaction between the P2 oligo (blue) and the single-stranded portion of the primer/template (green). The example uses template T50c and the P2 oligo 5'-GGCGCC-ext 3. See the legend of Figure 1 for more details. (B) Correlation between the predicted base-pairing stability and polymerization efficiency. The base-pairing stabilities between the P2 oligo 3'-extensions and the single-stranded portions of the primer/ templates were estimated by the UNAfold algorithm. All 23 P2 oligos with $60 \% \mathrm{G}$ and $40 \% \mathrm{~T}$ in their $3^{\prime}$-extension were used. Template T21 (open circles) resulted in a high polymerization efficiency with weak template/P2 oligo interactions, presumably because T21 interacts directly with the polymerase ribozyme. The linear regression was fitted to the values of templates T50a (blue circles), T50b (red triangles), and T50c (green squares). The linear regression coefficients $r$ are shown, together with $p$, which describes the likelihood that no correlation exists. For the three templates combined, the linear regression coefficient was $-0.575(p<0.0001)$.

In the mixture, each P2 oligo had a concentration of $1 / 3$ of $2.5 \mu \mathrm{M}$ so that the total concentration of P2 oligos was the same in the mixed and the separate reactions. This comparison was done on template T50c because this template showed a large difference between efficient and inefficient P2 oligos. When the P2 oligos were optimized for their efficiency on several different templates, we measured the average amount of nucleotides per primer (see above) and took the geometric mean of the result with the different template sequences. Sequence alignments used the software Clustal and were done as pairwise alignment followed by multiple sequence alignment, using the IUB DNA weight matrix.
To estimate the polymerization fidelity, we compared polymerization reactions in the presence of all NTPs with four polymerization reactions that each lacked one of the NTPs. The extension rate was determined as for the determination of $2^{\prime}$-deoxy effects (see below). The fold reduction $b$ of the extension rate by omitting a specific NTP was determined. This value $b$ served to calculate the polymerization fidelity with the equation fidelity = $100 \%(1-1 / b)$. The value $b$ could not be determined for each NTP and template due to limited polymerization efficiency, especially with the wild-type P2 oligo. The values were lower than previously reported fidelities for the same ribozyme (Johnston et al. 2001). This is probably a consequence of our fidelity assay, which monitored several nucleotide extensions, and because different template sequences were investigated.

Reactions testing the effect of 2'-deoxy modifications determined the rate of primer extension with the template T21 and compared it to the rates with the template T21 carrying a single $2^{\prime}$-deoxy substitution (5'-GACGCUdUCGCACGGUUGGCAG$\left.3^{\prime}\right)$. The data were processed based on a published procedure (Müller and Bartel 2003). In short, the fraction of primer with a specific length that reacted between reaction times $10 \mathrm{~min}$ and 60 min was determined. This fraction was used as a relative value for the primer extension rate. The value for the unmodified template was divided by the value for the template with the $2^{\prime}$ deoxy modification and served as the fold effect of the $2^{\prime}$-deoxy modification at one specific position. The position of the $2^{\prime}$-deoxy modification relative to the primer $3^{\prime}$-terminus changed with each nucleotide that was added to the primer $3^{\prime}$-terminus. Therefore, the position of the $2^{\prime}$-deoxy modification was deduced from the initial position of the $2^{\prime}$-deoxy modification $(+5)$ and the number of nucleotides that were added, for each intermediate. Because the 2 -deoxy modification changes its position relative to the primer 3 '-terminus during polymerization, it allowed the detection of $2^{\prime}$ deoxy effects at multiple positions relative to the primer $3^{\prime}$ terminus.

\section{SUPPLEMENTAL MATERIAL}

Supplemental material is available for this article.

\section{ACKNOWLEDGMENTS}

We thank Michael Lawrence and Matthew Jones-Rhoades for help in computationally designing the new template sequences. Simpson Joseph, Janina Moretti, Nav Toor, and Jing Yang are thanked for critical reading of the manuscript. This research was funded by NSF grant 0743985 to U.F.M.

Received March 15, 2011; accepted April 25, 2011.

\section{REFERENCES}

Chen X, Li N, Ellington AD. 2007. Ribozyme catalysis of metabolism in the RNA world. Chem Biodivers 4: 633-655.

Cheng LK, Unrau PJ. 2010. Closing the circle: Replicating RNA with RNA. Cold Spring Harb Perspect Biol 2: a002204. doi: 10.1101/ cshperspect.a002204.

Crick FHC. 1968. The origin of the genetic code. J Mol Biol 38: 367379.

Ekland EH, Bartel DP. 1995. The secondary structure and sequence optimization of an RNA ligase ribozyme. Nucleic Acids Res 23: 3231-3238. 
Ekland EH, Bartel DP. 1996. RNA-catalysed RNA polymerization using nucleoside triphosphates. Nature 382: 373-376.

Gilbert W. 1986. The RNA world. Nature 319: 618.

Huang W, Ferris JP. 2003. Synthesis of 35-40 mers of RNA oligomers from unblocked monomers. A simple approach to the RNA world. Chem Commun (Camb) 2003: 1458-1459.

Hunter WN, Brown T, Kneale G, Anand NN, Rabinovich D, Kennard O. 1987. The structure of guanosine-thymidine mismatches in B-DNA at 2.5-Å resolution. J Biol Chem 262: 99629970.

Johnston WK, Unrau PJ, Lawrence MS, Glasner ME, Bartel DP. 2001. RNA-catalyzed RNA polymerization: Accurate and general RNAtemplated primer extension. Science 292: 1319-1325.

Joyce GF. 2002. The antiquity of RNA-based evolution. Nature 418: 214-221.

Kalnik MW, Kouchakdjian M, Li BF, Swann PF, Patel DJ. 1988. Base pair mismatches and carcinogen-modified bases in DNA: An NMR study of A.C and A.O4meT pairing in dodecanucleotide duplexes. Biochemistry 27: 100-108.

Lawrence MS. 2005. "RNA polymerase ribozymes." $\mathrm{PhD}$ thesis, Massachusetts Institute of Technology, Cambridge, MA.
Lawrence MS, Bartel DP. 2003. Processivity of ribozyme-catalyzed RNA polymerization. Biochemistry 42: 8748-8755.

Lawrence MS, Bartel DP. 2005. New ligase-derived RNA polymerase ribozymes. RNA 11: 1173-1180.

Müller UF. 2006. Re-creating an RNA world. Cell Mol Life Sci 63: 1278-1293.

Müller UF, Bartel DP. 2003. Substrate 2' -hydroxyl groups required for ribozyme-catalyzed polymerization. Chem Biol 10: 799-806.

Orgel LE. 1968. Evolution of the genetic apparatus. J Mol Biol 38: 381393.

Shechner DM, Grant RA, Bagby SC, Koldobskaya Y, Piccirilli JA, Bartel DP. 2009. Crystal structure of the catalytic core of an RNApolymerase ribozyme. Science 326: 1271-1275.

Wang QS, Cheng LK, Unrau PJ. 2011. Characterization of the B6.61 polymerase ribozyme accessory domain. RNA 17: 469-477.

Wochner A, Attwater J, Coulson A, Holliger P. 2011. Ribozyme-catalyzed transcription of an active ribozyme. Science 332: 209-212.

Woese CR. 1967. The genetic code. The molecular basis for genetic expression. Harper \& Row, New York.

Zaher HS, Unrau PJ. 2007. Selection of an improved RNA polymerase ribozyme with superior extension and fidelity. RNA 13: 1017-1026. 

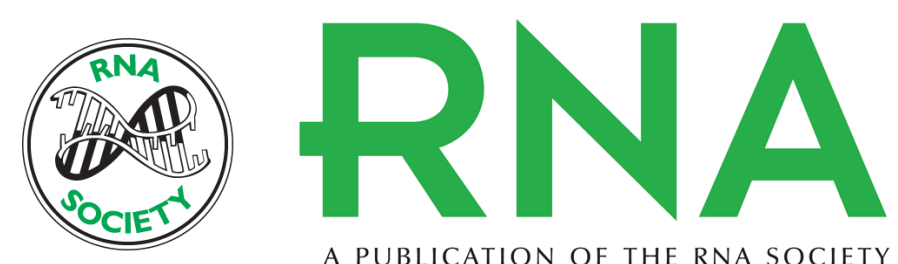

A PUBLICATION OF THE RNA SOCIETY

\section{Polymerase ribozyme efficiency increased by G/T-rich DNA oligonucleotides}

Chengguo Yao and Ulrich F. Müller

RNA 2011 17: 1274-1281 originally published online May 27, 2011

Access the most recent version at doi:10.1261/rna.2726811

\section{Supplemental http://rnajournal.cshlp.org/content/suppl/2011/05/13/rna.2726811.DC1 \\ Material}

References This article cites 20 articles, 8 of which can be accessed free at: http://rnajournal.cshlp.org/content/17/7/1274.full.html\#ref-list-1

\section{License}

Email Alerting Receive free email alerts when new articles cite this article - sign up in the box at the Service top right corner of the article or click here. 